\title{
Sonographic Evaluation for Endometrial Polyps
} The Interrupted Mucosa Sign

\author{
Aya Kamaya, MD, Pauline Chang Yu, MD, Carla Ramas Lloyd, MD, Bertha H. Chen, MD, \\ Terry S. Desser, MD, Katherine E. Maturen, MD
}

Received November 24, 2015, from the Departments of Radiology (A.K., T.S.D.) and Obstetrics and Gynecology (B.H.C.), Stanford University Medical Center, Stanford California USA; Department of Obstetrics and Gynecology, Kaiser Permanente, Santa Clara, CA (P.C.Y.); Virtual Radiologic Professionals, LLC, Eden Prairie, Minnesota USA (C.R.L.); and Department of Radiology, University of Michigan, Ann Arbor, Michigan USA (K.E.M.). Revision requested January 5, 2016. Revised manuscript accepted for publication February 6, 2016.

We thank Jeslyn A. Rumbold for editorial assistance.

Address correspondence to Aya Kamaya, $M D$, Department of Radiology, Stanford University Medical Center, 300 Pasteur Dr, H1307, Stanford, CA 94305 USA.

E-mail:kamaya@stanford.edu
Objectives - To evaluate the interrupted mucosa sign for identification of endometrial polyps, using pathologic confirmation as the reference standard, compared to other accepted sonographic findings.

Methods-We reviewed 195 patients referred for pelvic sonographic evaluations for suspected endometrial polyps in this retrospective Institutional Review Boardapproved study. Of these, 82 had tissue sampling of the endometrium and constituted the final study group. Patient data, including age, menopausal status, last menstrual period, and final pathologic diagnosis, were recorded. Sonograms were reviewed by 2 blinded board-certified radiologists for endometrial features, including thickness, echogenicity, vascularity, presence of a mass, and the interrupted mucosa sign. Descriptive statistics and multivariate logistic regression analysis were performed.

Results - The mean age of the patients was 44.99 (SD, 9.88) years, 79.1\% of whom were premenopausal. Pathologic diagnosis confirmed polyps in 58 (70.73\%). A single feeding vessel was visualized in 36 patients with polyps (62.07\%), whereas the interrupted mucosa sign was visualized in 34 (58.62\%). The presence of a feeding vessel, the interrupted mucosa sign, or both detected 48 (82.76\%) of the polyps. In the multivariate analysis, only the interrupted mucosa sign was a statistically significant predictor of pathologic diagnosis of a polyp $(P=.035)$, with an odds ratio of 3.83 ( $95 \%$ confidence interval, 1.10-13.29). Other sonographic findings were not independent predictors of a polyp: mass $(P=.35)$, single feeding vessel $(P=.31)$, endometrial thickness $(P=.88)$, and endometrial echogenicity $(P=.45)$. The sensitivity, specificity, and positive predictive value of the interrupted mucosa sign were $59 \%, 75 \%$, and $85 \%$, respectively.

Conclusions - The interrupted mucosa sign is a promising sonographic sign for identification of endometrial polyps, with greater predictive power than previously described signs. It has the potential to improve the diagnostic performance of sonography, especially when used in combination with other described signs.

Key Words — dysfunctional uterine bleeding; endometrial polyp; feeding vessel; gynecologic ultrasound; interrupted mucosa; pedicle artery sign

E ndometrial polyps are benign focal overgrowths of endometrial tissue, often with variable amounts of vascularity and stroma encased by epithelium. Although the etiology of polyp development is unclear, endometrial polyps are quite common, identified in $13 \%$ to $50 \%$ of women with dysfunctional uterine bleeding and $10 \%$ of asymptomatic patients undergoing routine sonographic imaging. ${ }^{1}$ Standard treatment is hysteroscopic removal to alleviate bleeding symptoms and exclude malignancy. ${ }^{2}$ 
Typically, patients presenting with dysfunctional uterine bleeding are first assessed by pelvic sonography. Depending on sonographic findings, patients may be referred for further evaluation with saline-infused sonohysterography or hysteroscopic removal. The most widely accepted and commonly used sonographic features of a polyp are an echogenic endometrial lesion with a single feeding vessel. ${ }^{3-5}$ Although these findings are extremely helpful, they are not always sonographically evident, and visualization may depend on body habitus or timing of imaging during the phase of menstrual cycle (Figure 1). ${ }^{6}$ In our clinical practice, we have observed an additional sonographic finding: the interrupted mucosa sign, which may help in the diagnosis of endometrial polyps. The interrupted mucosa sign is identified when the highly echogenic linear interface where opposing endometrial mucosal surfaces coapt can be followed to a point at which it is focally interrupted (typically by an endometrial polyp). In our experience, the focal interruption is usually best appreciated on transvaginal transverse or longitudinal images of the uterus during the first half of the menstrual cycle (Figure 2). The interrupted mucosa sign may also be helpful during the latter half of the menstrual cycle, when polyps may be isoechoic to the endometrium and their borders indistinct (Figures 3 and 4). The purpose of our study was to investigate the interrupted mucosa sign in the diagnosis of endometrial polyps.

\section{Materials and Methods}

We reviewed 195 patients referred for sonographic evaluations of the female pelvis performed at out institution in this retrospective Institutional Review Board-approved study using the key word search term "polyp" in pelvic sonographic reports. Informed consent was waived by the Institutional Review Board. Of these patients, 82 had pathologic confirmation of endometrial findings, and these patients constituted our final study group. Patient data, including age, menopausal status, last menstrual period, and final pathologic diagnosis, were recorded. If the last menstrual period date was not provided, the age of 50 years or older was used as a threshold for categorization as menopausal.

All sonographic examinations were performed by American Registry for Diagnostic Medical Sonographycertified sonographers under the supervision of a boardcertified radiologist. All examinations were performed on either Acuson Sequoia 512 (Siemens Medical Solutions, Mountain View, CA) or LOGIQ E9 (GE Healthcare, Waukesha, WI) ultrasound machines. Transabdominal images were typically obtained with a full bladder at 36-MHz frequencies. Patients were then asked to empty their bladder, and transvaginal images were obtained of the uterus at $6-8-\mathrm{MHz}$ frequencies with grayscale and color Doppler imaging. Power and spectral Doppler evaluations were used as deemed appropriate by the sonographer or interpreting radiologist at the time of the examination, for example, to provide greater sensitivity for detection of focal vascularity or to characterize detected focal vascularity as arterial or venous.

Figure 1. Interrupted mucosa sign without a feeding vessel. An echogenic polyp (blue arrow) is shown, which focally interrupts the endometrial mucosa (yellow arrows). However, a feeding vessel was not visualized on color Doppler imaging.

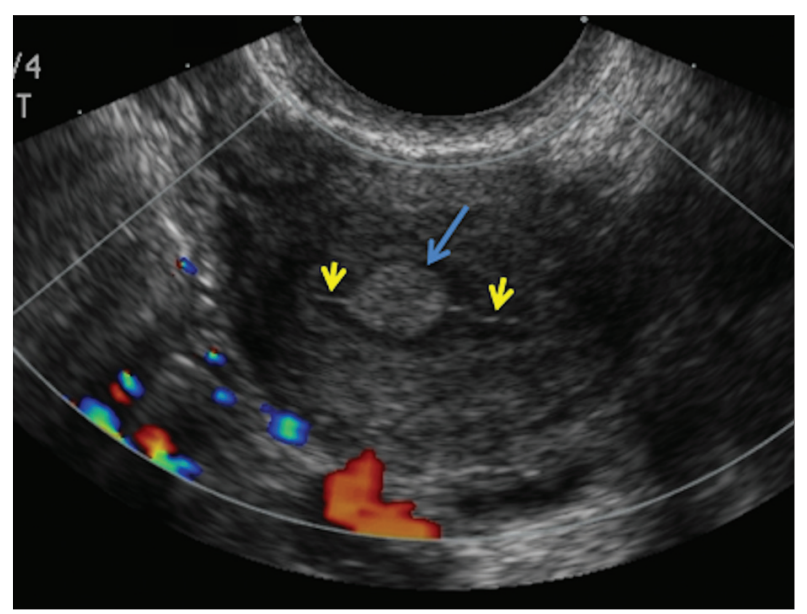

Figure 2. Interrupted mucosa sign in a hypoechoic endometrial echo complex. The mucosa is often best visualized during the first half of the menstrual cycle, when the endometrium is hypoechoic in appearance. In this example, the endometrial-mucosal interface (yellow arrows) is clearly interrupted by a polyp (blue arrow).

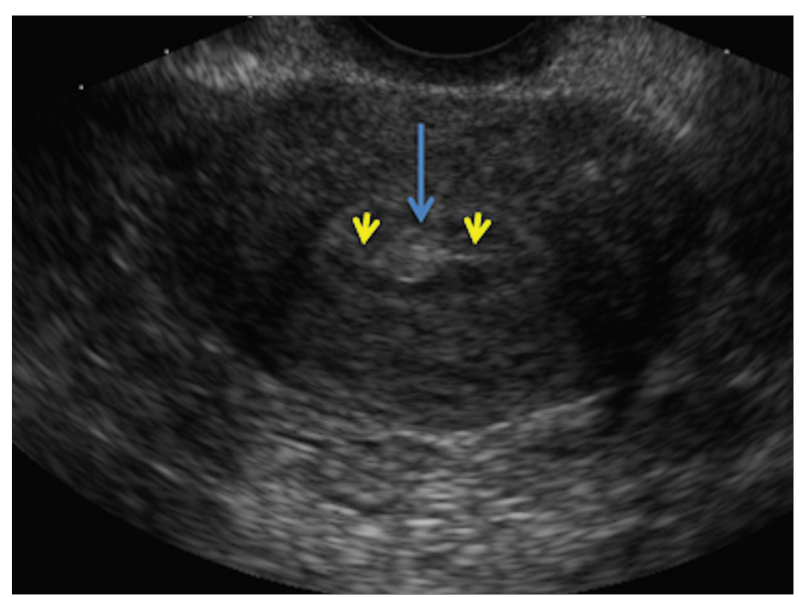


Sonograms were retrospectively reviewed in consensus by 2 board-certified radiologists (A.K. and C.R.L.) for the presence of the interrupted mucosa sign, sonographic visibility of an endometrial mass, presence of a single feeding vessel versus multiple vessels, and endometrial appearance and thickness. The endometrial appearance was assessed as hypoechoic, isoechoic, or echogenic compared to the adjacent myometrium. Endometrial thickness was measured at its maximum anteroposterior thickness viewed in the sagittal plane with one caliper placed in the anterior uterine wall at the margin of the basal layer of the endometrium and the other caliper placed at the same margin in the posterior uterine wall, excluding fluid (if present) in the endometrial cavity. ${ }^{7}$ Radiologists were blinded to the final pathologic diagnoses. Images, including still images and cine clips (when available) were reviewed on Syngo Kinetdx workstations (Siemens Medical Solutions).

Descriptive statistical analyses were performed using means, counts, and percentages, and comparisons were made between the polyp and nonpolyp groups by a $t$ test for continuous variables, and a $\chi^{2}$ or Fisher exact test for categorical variables. Multivariate logistic regression for prediction of the presence of polyps on pathologic examination was performed with automated forward selection of variables, with $P=.05$ used as the inclusion criterion. Age, menopausal status, and history of hormone replacement therapy were included as a priori predictors. Imaging findings, including the presence, size, echogenicity (hypoechoic, isoechoic, or echogenic), and vascularity

Figure 3. Interrupted mucosa sign. A subtle endometrial polyp (blue arrow) is shown in an echogenic endometrial echo complex. The echogenic mucosa (yellow arrows) can be followed to the level of the polyp, which focally interrupts the mucosal line. Without careful evaluation of the mucosa, the echogenic polyp may otherwise be difficult to detect in a background of echogenic endometrium.

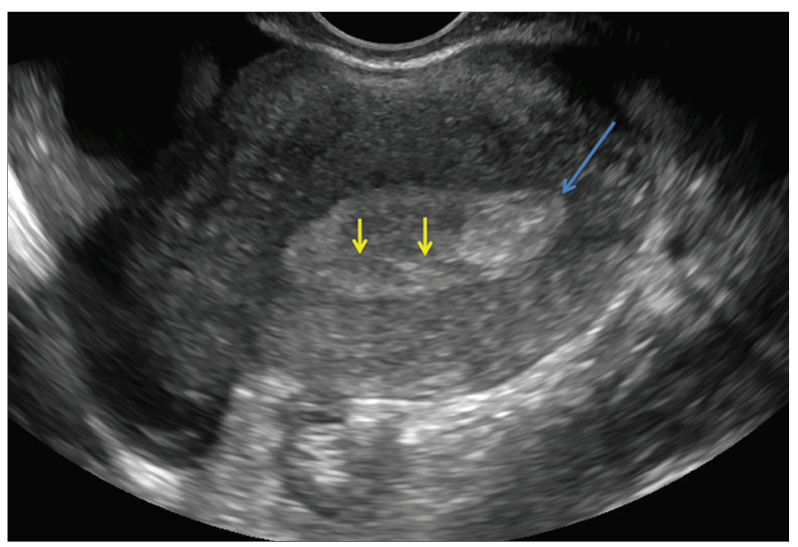

(single or multiple feeding vessels) of a sonographic mass, the interrupted mucosa sign, and interaction terms including "menopause*interrupted sign" and "menopause* single feeding vessel" were entered into automated forward selection of variables. The diagnostic performance (sensitivity, specificity, positive predictive value, and negative predictive value) of the interrupted mucosa sign was evaluated by using pathologic confirmation as the reference standard. All analyses were performed with SAS version 9.3 software (SAS Institute Inc, Cary, NC).

Figure 4. Interrupted mucosa sign without an associated mass in a pathologically proven 2-cm polyp. A, Grayscale transvaginal sonogram showing that the mucosa (yellow arrows) is focally interrupted (blue arrow), without a clear sonographically visible associated mass. B, Color Doppler sonogram from the same patient showing a vessel in the endometrium, which terminates as the point of the focal interruption (yellow arrow) of the endometrium.
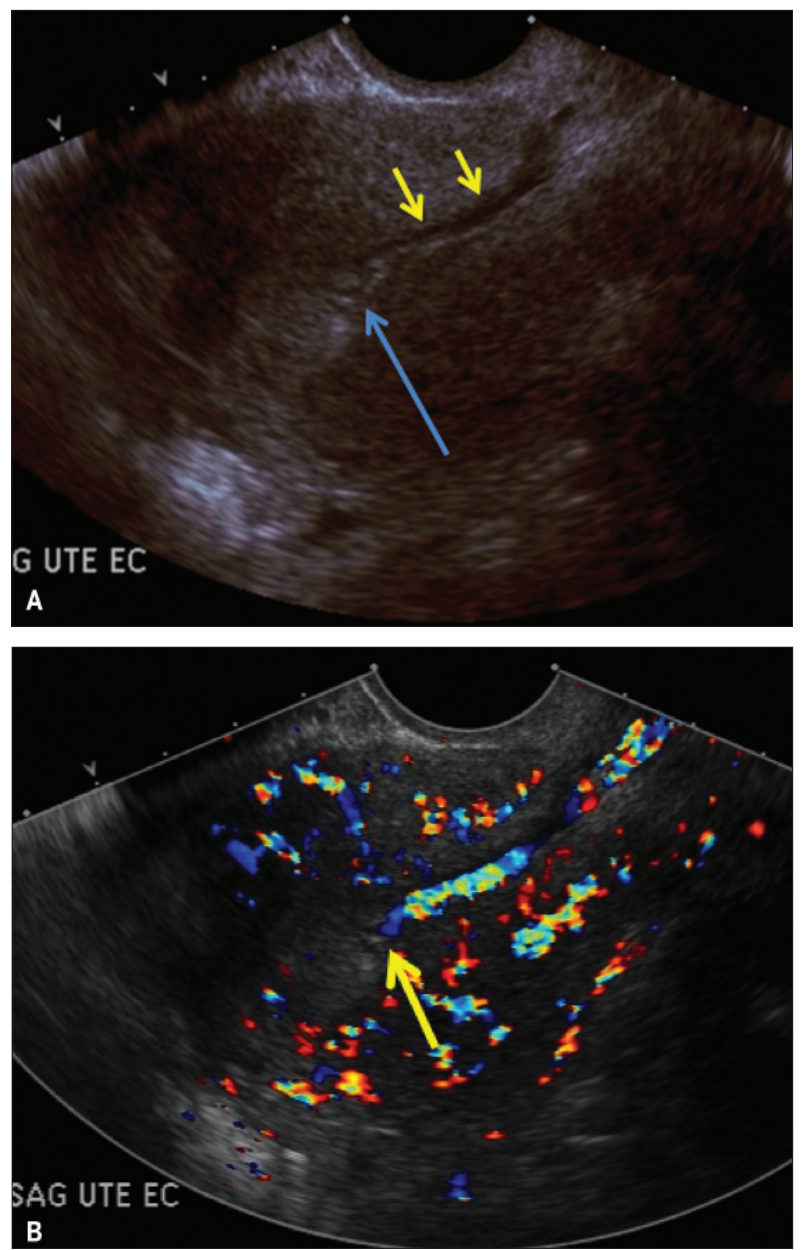


\section{Results}

The study population consisted of 82 women, $79.1 \%$ of whom were premenopausal. Patient demographics are outlined in Table 1. The final pathologic diagnosis confirmed leiomyoma in $6(7.32 \%)$ women, endometrial hyperplasia in 2 (2.44\%), polyps in 58 (70.73\%), and no abnormality in 16 (19.51\%). Pathologic confirmation was obtained by hysteroscopy in 72 patients, total hysterectomy in 5, and endometrial biopsy in 5 .

The sonographic findings are enumerated in Table 2. In the univariate analysis, the detection frequency of a sonographic mass and its size, echogenicity, and vascularity did not differ significantly between the polyp and nonpolyp groups. The interrupted mucosa sign was much more frequently present among polyps. A single feeding vessel was identified in more patients with polyps than in patients without polyps.
The presence of a feeding vessel, the interrupted mucosa sign, or both detected 48 (82.76\%) of the polyps; $22(38.00 \%)$ had both a feeding vessel and interrupted mucosa, and $26(45.00 \%)$ had either a feeding vessel or interrupted mucosa. In the evaluation of a mass versus the interrupted mucosa sign, in patients with polyps, 30 of $58(51.72 \%)$ had both a mass and the interrupted mucosa sign; 7 (12.07\%) did not have a mass or the interrupted mucosa sign; 18 (31.03\%) had a mass but no interrupted mucosa sign; and $3(5.17 \%)$ had the interrupted mucosa sign but no mass. Of the patients without polyps, 6 of $24(25.00 \%)$ had no mass or interrupted mucosa sign; 13 (54.17\%) had a mass but no interrupted mucosa sign; 5 (20.83\%) had both the interrupted mucosa sign and a mass; and 0 had the interrupted mucosa sign but no mass.

Table 1. Clinical Characteristics of 82 Patients With Clinically Suspected Endometrial Polyps

\begin{tabular}{lcccc}
\hline Characteristic & Total & $\begin{array}{c}\text { Pathologic Diagnosis: } \\
\text { + Polyp }\end{array}$ & $\begin{array}{c}\text { Pathologic Diagnosis: } \\
\text { - Polyp }\end{array}$ & P \\
\hline Patients, $\mathrm{n}$ & 82 & 58 & 24 & $\mathrm{NA}$ \\
Mean age, $\mathrm{y}(\mathrm{SD})$ & $44.99(9.88)$ & $44.65(10.82)$ & $45.79(7.21)$ & .63 \\
Postmenopausal, $\mathrm{n}(\%)$ & $17(20.99)$ & $12(20.83)$ & $5(21.05)$ & .98 \\
Hormone replacement therapy, $\mathrm{n}(\%)$ & $7(8.64)$ & $5(8.33)$ & $2(8.77)$ & $>.99 \mathrm{a})$ \\
\hline
\end{tabular}

NA indicates not applicable.

aFisher exact test used for comparison because of low cell count.

Table 2. Univariate Analysis of Imaging Features of Endometrial Polyps Versus Other Pathologic Diagnoses, Including Leiomyoma, Hyperplasia, and No Pathologic Abnormality

\begin{tabular}{|c|c|c|c|c|}
\hline Characteristic & Total & $\begin{array}{c}\text { Pathologic Diagnosis: } \\
\text { + Polyp }\end{array}$ & $\begin{array}{c}\text { Pathologic Diagnosis: } \\
\text { - Polyp }\end{array}$ & $P$ \\
\hline Mass shown on sonography, n (\%) & $67(81.71)$ & $49(84.48)$ & $18(75.00)$ & $.35^{a}$ \\
\hline Mean longest mass dimension, mm (SD) & $11.64(7.96)$ & $11.14(5.39)$ & $13.00(12.69)$ & .40 \\
\hline Mean mass area, $\mathrm{mm}^{2}(\mathrm{SD})^{\mathrm{b}}$ & $128.69(178.36)$ & $114.30(119.10)$ & $167.8(285.00)$ & .28 \\
\hline Echogenic compared to endometrium, $\mathrm{n}(\%)$ & $28(41.79)$ & $23(46.94)$ & $5(27.78)$ & .10 \\
\hline Isoechoic compared to endometrium, n (\%) & $28(41.79)$ & $18(36.73)$ & $10(55.56)$ & .36 \\
\hline Hypoechoic compared to endometrium, n (\%) & $11(16.42)$ & $8(16.33)$ & $3(16.67)$ & $>.99^{a}$ \\
\hline Vascularity, $\mathrm{n}(\%)$ & $74(90.24)$ & $52(89.66)$ & $22(92.67)$ & $>.99^{a}$ \\
\hline Single feeding vessel, n (\%) & $48(58.54)$ & $36(62.07)$ & $12(50.00)$ & .31 \\
\hline$>1$ feeding vessel, $n(\%)$ & $12(14.63)$ & $6(10.34)$ & $6(25.00)$ & $.10^{a}$ \\
\hline Interrupted mucosa sign, $\mathrm{n}(\%)$ & $40(48.78)$ & $34(58.62)$ & $6(25.00)$ & .006 \\
\hline Mean endometrial thickness, mm (SD) & $10.75(5.06)$ & $10.81(5.24)$ & $10.62(4.67)$ & .88 \\
\hline Echogenic endometrium, n (\%) & $46(56.10)$ & $31(37.80)$ & $15(62.50)$ & .45 \\
\hline
\end{tabular}

aFisher exact test used because of low cell counts.

${ }^{b}$ Calculated as an ellipse based on cross-sectional measurements in a sagittal view. 
The sensitivity, specificity, positive predictive value, and negative predictive value of the interrupted mucosa sign were $59 \%, 75 \%, 85 \%$, and $43 \%$, respectively (Figures 5-7). For a single feeding vessel, these values were $62 \%, 50 \%$, $75 \%$, and $50 \%$, and for the presence of a mass, the values were $84 \%, 25 \%, 73 \%$, and $40 \%$.

In the multivariate logistic regression, only the interrupted mucosa sign was retained as a statistically significant predictor of pathologic diagnosis of polyp. There was no statistically significant interaction effect $(P>.05)$ between menopause status and the interrupted mucosa sign or menopause and the presence of a single feeding vessel as imaging predictors of the polyp outcome. Parameter estimates and results of the logistic regression analysis are given in Table 3.

\section{Discussion}

The sonographic diagnosis of an endometrial polyp remains clinically challenging. Indeed, most imaging features on which radiologists rely were either not predictive or simply may not have reached the threshold of significance for pathologic diagnosis of polyps in our study, including echogenicity, vascularity, size of a mass, and even sonographic detection of a mass. However, we describe a new imaging feature: the interrupted mucosa sign, which was the only statistically significant predictor of pathologic diagnosis of a polyp in the multivariate logistic regression analysis.

Figure 5. False-positive interrupted mucosa sign. An echogenic mass (blue arrow) is shown, which focally interrupts the mucosal line (yellow arrows). However, on blind endometrial biopsy, pathologic examination only showed a proliferative endometrium, and no polyp was identified.

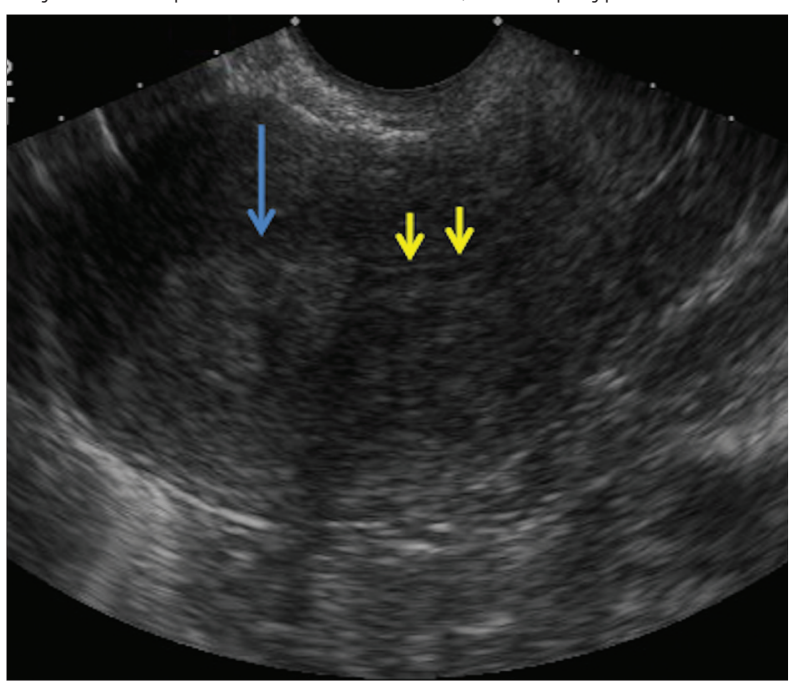

We believe that this sonographic finding is related to the underlying pathologic nature of endometrial polyps itself. These polyps are focal overgrowths of the endometrium; thus, their origin is within the endometrium. An endometrial polyp would therefore focally interrupt the normal mucosal contour of the uterine cavity. In contrast,

Figure 6. False-positive interrupted mucosa sign. A, An echogenic mass (blue arrows) is shown, which focally interrupts the mucosal line (yellow arrows). B, T2 weighted magnetic resonance image of the uterus showing that the mass (blue arrow) is a submucosal fibroid.
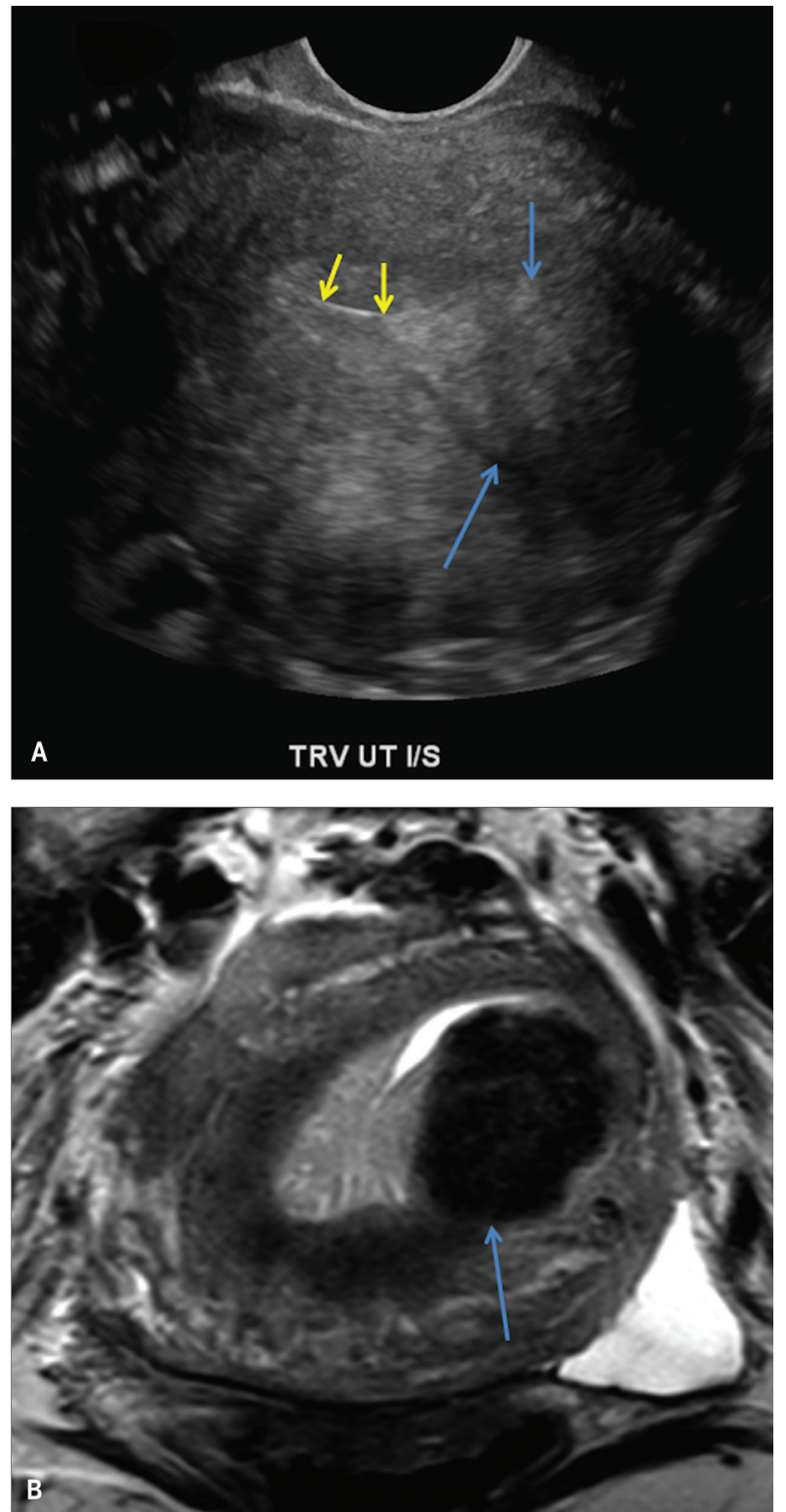
fibroids arise from the myometrium and characteristically have a covering layer of endometrium. A submucosal fibroid, therefore, would not typically interrupt the endometrial mucosa. As with other sonographic findings, the interrupted mucosa sign is more likely to be visualized during the first half of the menstrual cycle, when the endometrium is thinner and relatively hypoechoic.

If an endometrial polyp is suspected, patients are often further evaluated with saline-infused sonohysterography when the pelvic sonogram shows an abnormality. Although saline-infused sonohysterography can be helpful in further delineating the endometrium, it has several potential drawbacks: first, the technique may cause more discomfort and higher cost to patients; second, conditions such as cervical stenosis may preclude such an evaluation ${ }^{8}$; and third, some studies have raised the concern that salineinfused sonohysterography can lead to intraperitoneal spillage of malignant cells with unsuspected endometrial malignancy. ${ }^{9}$ Therefore, if a polyp can be confidently diag-

Figure 7. False-negative interrupted mucosa sign. An isoechoic mass (blue arrows) is shown abutting and distorting but not interrupting the mucosal line (yellow arrows) in this patient, who was confirmed to have a polyp on pathologic examination.

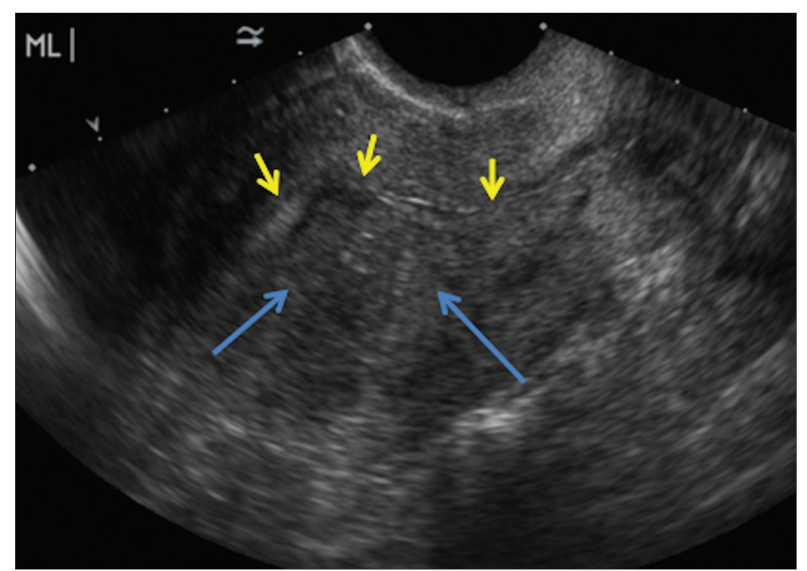

nosed on conventional sonography, those patients may be directly treated with hysteroscopic removal and avoid saline-infused sonohysterography. We think that the additional sonographic finding of an interrupted mucosa can help in triaging patients directly toward hysteroscopic removal of an endometrial polyp and avoiding confirmatory but nontherapeutic saline-infused sonohysterography.

Several previous studies have compared the sensitivity of routine pelvic sonography to saline-infused sonohysterography for identification of endometrial polyps. ${ }^{10-13}$ However, many of these studies only included patients with findings on pelvic sonography, which were then further characterized by saline-infused sonohysterography, leading to a selection bias and apparently improved detection rates for saline-infused sonohysterography. In fact, the standard pelvic sonographic examination is extremely important in this chain of evaluation, yet there are very few studies in the literature that have looked solely at the features of endometrial polyps on conventional pelvic sonography. The most commonly cited sonographic feature is the pedicle artery sign, described by Timmerman et $\mathrm{al}^{3}$ in 2003, in which a single feeding vessel is identified on color Doppler sonography. In a slightly older publication by Baldwin et al, ${ }^{14}$ a hyperechoic line along the periphery of the endometrial cavity was found to be a good predictor of fibroids, polyps, hyperplasia, and localized neoplasms. However, in that study, this sign was not used to distinguish among these entities.

There were several limitations to our study. First, it was a retrospective evaluation, and the initial patient list was selected on the basis of a clinical or sonographic suspicion of an endometrial polyp, which led to an artificially high prevalence of endometrial polyps that is not reflective of the general population in clinical practice and limited evaluation of the true sensitivity and specificity of the sign. Therefore, these estimates must be interpreted with caution. In addition, clinically silent endometrial polyps may not constitute a proportional subset of the study group; however, because we required pathologic confirmation of

Table 3. Results and Parameter Estimates From Multivariate Logistic Regression Analysis of Clinical and Imaging Features Predictive of Pathologic Diagnosis of a Polyp

\begin{tabular}{lcccc}
\hline Parameter & Estimate & SE & $\boldsymbol{P}$ & OR (95\% Cl) \\
\hline Intercept & 2.15 & 2.12 & .311 & NA \\
Age & -0.05 & 0.05 & .335 & $0.95(0.87-1.05)$ \\
Postmenopausal & 0.97 & 1.15 & .399 & $2.63(0.28-25.03)$ \\
Hormone replacement therapy & 0.76 & 1.27 & .541 & $2.17(0.18-26.11)$ \\
Interrupted mucosa sign & 1.34 & 0.64 & .035 & $3.83(1.10-13.29)$ \\
\hline
\end{tabular}

$\mathrm{Cl}$ indicates confidence interval; NA, not applicable; and OR, odds ratio. 
endometrial findings, this selection bias was unavoidable. The second limitation of our study was that the patient cohort was relatively small; because we maintained a rigorous standard requiring definitive tissue diagnosis of endometrial contents, this approach limited the number of patients in our study cohort. A third limitation was that there were no cancers identified in our study group. However, the expected incidence of cancer in our study population is relatively low $(0.5 \%-3 \%),{ }^{15-17}$ and other studies have found few if any endometrial cancers in their populations of patients with abnormal vaginal bleeding., 3,12 Nonetheless, we do not have the needed data to analyze whether the interrupted mucosa sign is helpful in differentiating endometrial polyps from cancers. A fourth limitation of our study was that, although all patients had pathologic correlation, 5 of the 82 patients only had blind endometrial biopsy, and it is possible that on blind endometrial biopsy, an endometrial polyp identified by sonography could potentially be missed (Figure 5). Another limitation was that distinguishing focal interruption from displacement from a submucosal fibroid may in some cases be difficult, potentially leading to false-positive results (Figure 6). Finally, we do not know how often the mucosal line is simply not visualized in some individuals, in whom the usefulness of this finding would be limited.

In conclusion, the interrupted mucosa sign is a promising sonographic feature for identification of endometrial polyps and could contribute to patient care by directing patients to prompt hysteroscopy, thereby potentially avoiding intermediate invasive confirmatory tests such as saline-infused sonohysterography.

\section{References}

1. Clevenger-Hoeft M, Syrop CH, Stovall DW, VanVoorhis BJ. Sonohysterography in premenopausal women with and without abnormal bleeding. Obstet Gynecol 1999; 94:516-520.

2. Uglietti A, Mazzei C, Deminico N, Somigliana E, Vercellini P, Fedele L. Endometrial polyps detected at ultrasound and rate of malignancy. Arch Gynecol Obstet 2014; 289:839-843.

3. Timmerman D, Verguts J, Konstantinovic ML, et al. The pedicle artery sign based on sonography with color Doppler imaging can replace second-stage tests in women with abnormal vaginal bleeding. Ultrasound Obstet Gynecol 2003; 22:166-171.

4. Tamura-Sadamori R, Emoto M, Naganuma Y, Hachisuga T, Kawarabayashi T. The sonohysterographic difference in submucosal uterine fibroids and endometrial polyps treated by hysteroscopic surgery. JUltrasound Med 2007; 26:941-946.

5. Kupfer MC, Schiller VL, Hansen GC, Tessler FN. Transvaginal sonographic evaluation of endometrial polyps. JUltrasound Med 1994; 13:535-
539.

6. Jokubkiene L, Sladkevicius P, Valentin L. Appearance of the endometrium at saline contrast sonohysterography in the luteal phase of the menstrual cycle: a prospective observational study. Ultrasound Obstet Gynecol 2015; 45:339-345.

7. Bredella MA, Feldstein VA, Filly RA, Goldstein RB, Callen PW, Genant HK. Measurement of endometrial thickness at US in multicenter drug trials: value of central quality assurance reading. Radiology 2000; 217:516520.

8. Verrotti C, Benassi G, Caforio E, Nardelli GB. Targeted and tailored diagnostic strategies in women with perimenopausal bleeding: advantages of the sonohysterographic approach. Acta Biomed 2008; 79:133-136.

9. Zerbe MJ, Zhang J, Bristow RE, Grumbine FC, Abularach S, Montz FJ. Retrograde seeding of malignant cells during hysteroscopy in presumed early endometrial cancer. Gynecol Oncol 2000; 79:55-58.

10. Grimbizis GF, Tsolakidis D, Mikos T, et al. A prospective comparison of transvaginal ultrasound, saline infusion sonohysterography, and diagnostic hysteroscopy in the evaluation of endometrial pathology. Fertil Steril 2010; 94:2720-2725.

11. Soguktas S, Cogendez E, Kayatas SE, Asoglu MR, Selcuk S, Ertekin A. Comparison of saline infusion sonohysterography and hysteroscopy in diagnosis of premenopausal women with abnormal uterine bleeding. Eur J Obstet Gynecol Reprod Biol 2012; 161:66-70.

12. AlcázarJL, Galan MJ, MínguezJA, García-Manero M. Transvaginal color Doppler sonography versus sonohysterography in the diagnosis of endometrial polyps. J Ultrasound Med 2004; 23:743-748.

13. La Sala GB, Blasi I, Gallinelli A, et al. Diagnostic accuracy of sonohysterography and transvaginal sonography as compared with hysteroscopy and endometrial biopsy: a prospective study. Minerva Ginecol 2011; 63:421-427.

14. Baldwin MT, Dudiak KM, Gorman B, Marks CA. Focal intracavitary masses recognized with the hyperechoic line sign at endovaginal US and characterized with hysterosonography. Radiographics 1999; 19:927-935.

15. Goldstein SR, Monteagudo A, Popiolek D, Mayberry P, Timor-Tritsch I. Evaluation of endometrial polyps. Am J Obstet Gynecol 2002; 186:669_ 674.

16. Jakab A, Ovári L, Juhász B, Birinyi L, Bacskó G, Tóth Z. Detection offeeding artery improves the ultrasound of endometrial polyps in asymptomatic patients. Eur J Obstet Gynecol Reprod Biol 2005; 119:103-109.

17. Machtinger R, Korach J, Padoa A, et al. Transvaginal ultrasound and diagnostic hysteroscopy as a predictor of endometrial polyps: risk factors for premalignancy and malignancy. Int J Gynecol Cancer 2005; 15:325-328. 